\title{
Visfatin levels are low in subjects with type 2 diabetes compared to age-matched controls
}

\author{
Subhashini Yaturu*, Jared Davis², LaTonya Franklin², Runhua Shi ${ }^{3}$, Prasanna Venkatesh ${ }^{4}$, \\ Sushil K. Jain ${ }^{3}$ \\ ${ }^{1}$ Department of Medicine, Stratton VA Medical Center/AMC, Albany, USA; \\ *Corresponding Author: Subhashini.Yaturu@va.gov, yaturu@yahoo.com \\ ${ }^{2}$ Department of Research, Overton Brooks VA Medical Center, Shreveport, USA \\ ${ }^{3}$ Louisiana State University Health Sciences Center, Shreveport, USA \\ ${ }^{4}$ Overton Brooks VA Medical Center/LSUHSC, Shreveport, USA
}

Received 6 September 2012; revised 8 October 2012; accepted 19 October 2012

\begin{abstract}
Background: Visceral adiposity correlates strongly with insulin resistance and components of metabolic syndrome. Visfatin is preferentially produced by human visceral adipose tissue. Objective: The objective of this study was to evaluate the relationship between circulating levels of visfatin and other adipocytokines in patients with type 2 diabetes (DM) compared to the age-matched control subjects without DM. Methods: Anthropometric parameters, lipid profiles, high-sensitivity CRP (hs-CRP), insulin resistance [HOMA-IR], and levels of visfatin, TNF$\alpha$, and resistin were evaluated in 55 subjects with diabetes and 56 age matched control subjects without diabetes. Results: The visfatin levels were significantly lower in subjects with diabetes $(p<0.0001)$. Visfatin levels correlated strongly with resistin and CRP. We noted negative correlation of visfatin levels with BMI, triglycerides and glucose. Conclusion: These data suggest that visfatin may be a marker of subclinical inflammatory state.
\end{abstract}

Keywords: Visfatin; Adiponectin; Resistin; Diabetes; Insulin Resistance; TNF- $\alpha$; BMI; Metabolic

Syndrome; Visceral Adiposity

\section{INTRODUCTION}

Obesity is associated with an array of health problems including insulin resistance, type 2 diabetes, and atherosclerosis. Adipocytokines (also known as adipokines) are cytokines secreted by adipocytes. Cytokines are immunomodulating agents. Visfatin, also known as pre-B-cell colony-enhancing factor (PBEF) [1] and nicotinamide phosphoribosyltransferase (Nampt), a 52-kDa protein, is secreted from visceral fat. Visfatin has a unique structure and no obvious homology to other known proteins [2]. Visfatin is reported to exert insulin-mimetic effects in cultured cells and to lower plasma glucose levels in mice by binding to and activating the insulin receptor [3]. The biology of PBEF/visfatin is complex and many key questions remain unanswered, with emerging knowledge [2]. There are controversies regarding the association of visfatin with overweight/obesity, type 2 diabetes mellitus and insulin resistance (IR) in published literature. This study aimed to evaluate the visfatin levels in subjects with type 2 diabetes compared to age-matched subjects without diabetes and their relation to the clinical and biochemical parameters as well as some inflammatory cytokines.

\section{RESEARCH DESIGN AND METHODS}

The study was conducted in accordance with the Declaration of Helsinki and was approved by the Institutional Review Board at LSUHSC and Research and Development Committee at Overton Brooks VA Medical Center. Subjects with diabetes were originally recruited for the evaluation of the effect of pioglitazone on bone mineral density. The study subjects include 55 subjects with diabetes and 56 age-matched control subjects without diabetes. Subjects with diabetes were on pioglitazone for 1 - 3 months prior to the collection of the samples. The clinical and demographic data obtained from subjects during clinical evaluation, along with lipid parameters, are given in Tables 1 and 2. The clinical details include blood pressure, height, weight, BMI, measurement of waist circumference, smoking, alcohol use, and family history of diabetes.

Exclusion criteria included subjects with diseases known to be associated with disordered glucose metabo- 
Table 1. Clinical characteristics of the subjects with or without diabetes. Data expressed as \pm SD; BMI: body mass index.

\begin{tabular}{cccc}
\hline & $\begin{array}{c}\text { Diabetes } \\
(\mathbf{n}=\mathbf{5 5})\end{array}$ & $\begin{array}{c}\text { No } \\
\text { diabetes } \\
(\mathbf{n}=\mathbf{5 6})\end{array}$ & $\boldsymbol{p}$-value \\
\hline Age (yr) & $56 \pm 1.5$ & $56 \pm 1.6$ & 1 \\
BMI (kg/m $\left.\mathbf{m}^{2}\right)$ & $33.4 \pm 0.74$ & $27.9 \pm 0.6$ & $<0.001$ \\
Hypertension (\%) & 86 & 74 & NS \\
CAD (\%) & 39 & 36 & 0.7 \\
\hline
\end{tabular}

Table 2. Biochemical characteristics of the subjects with or without diabetes. Data expressed as $\pm \mathrm{SD} ; p<0.05$ is significant; HOMA-IR: Homeostasis model assessment of insulin resistance, T. Chol: Total cholesterol; F. Glucose: Fasting glucose.

\begin{tabular}{cccc}
\hline & $\begin{array}{c}\text { Diabetes } \\
(\mathbf{n}=\mathbf{5 5})\end{array}$ & $\begin{array}{c}\text { No } \\
\text { diabetes } \\
(\mathbf{n}=\mathbf{5 6})\end{array}$ & $\boldsymbol{p}$-value \\
\hline Creatinine & $1.09 \pm 0.03$ & $1.19 \pm 0.06$ & NS \\
F. Glucose (mg/dL) & $167 \pm 9.8$ & $98 \pm 2.0$ & $<0.0001$ \\
Fasting insulin & $18.8 \pm 3.2$ & $4.9 \pm 0.52$ & $<0.0001$ \\
HOMA-R & $66.5 \pm 16$ & $17.26 \pm 2.5$ & $<0.0001$ \\
T. Chol (mg/dL) & $161 \pm 4.9$ & $177 \pm 5.3$ & $<0.001$ \\
HDL-C (mg/dL) & $41 \pm 1.6$ & $47 \pm 1.8$ & 0.004 \\
LDL-C (mg/dL) & $90 \pm 4.3$ & $106 \pm 4.8$ & 0.004 \\
Triglycerides & $156 \pm 12$ & $120 \pm 5.7$ & NS \\
\hline
\end{tabular}

lism, such as Cushing's disease, acromegaly, pheochromocytoma, chronic pancreatitis, pancreatectomy, dumping syndrome, sub-optimally treated thyroid disease, subjects on medications that alter glucose metabolism, including glucocorticoids, pentamidine, nicotinic acid, diazoxide, $\beta$-adrenergic agonists, thiazides, dilantin, interferon, retroviral agents and anti-neoplasic agents; and subjects with chronic kidney disease .

\section{COLLECTION OF SAMPLES}

Blood samples were obtained in the morning after an overnight fast $(\sim 10 \mathrm{~h})$. For each subject, a basal metabolic panel, $\mathrm{HbA}_{1 \mathrm{C}}$ and lipid panel were analyzed in the central chemistry laboratory. Plasma was isolated from fresh blood and stored at $-80^{\circ} \mathrm{C}$ until further analysis for insulin, Visfatin, CRP, resistin and adiponectin.

\section{ASSAY METHODS}

Serum levels of visfatin, insulin, adiponectin, resistin, and high sensitivity C-reactive protein (CRP) were measured by enzyme-linked immunosorbent assay (ELISA); glucose and lipid profiles were measured using colorimetric enzymatic methods. Insulin resistance was measured using Homeostasis Model Assessment-Insulin Resistance (HOMA-IR). Levels of total adiponectin, resistin, TNF- $\alpha$, CRP and insulin were measured in duplicate using commercially available enzyme-linked immunosorbent assay (ELISA) kits (ALPCO Diagnostics, Salem, NH, USA). Visfatin (Nampt/PBEF) levels were measured in serum by ELISA using kits from ALPCO. The sensitivity of the visfatin assay is $30 \mathrm{pg} / \mathrm{mL}$. Interand intra-assay coefficients of variation were $3.8 \%$ and $6 \%$, respectively. The sensitivity of the adiponectin assay is $0.234 \mathrm{ng} / \mathrm{mL}$ and a range of 0.375 and $12 \mathrm{ng} / \mathrm{mL}$. Interand intra-assay coefficients of variation were $2.8 \%$ $5.5 \%$ and $2.97 \%-3.84 \%$, respectively. The sensitivity of the resistin assay is $0.2 \mathrm{ng} / \mathrm{mL}$. Inter-and intra-assay coefficients of variation were $4.2 \%-7.2 \%$ and $2.8 \%-5.1 \%$, respectively. The sensitivity of the CRP assay is 0.124 $\mathrm{ng} / \mathrm{mL}$. Inter-and intra-assay coefficients of variation were $5.5 \%-6 \%$ and $11 \%$ - 13\%, respectively. The sensitivity of the insulin assay is $1 \mathrm{IU} / \mathrm{mL}$. Inter- and intra-assay coefficients of variation were $2.6 \%-3.6 \%$ and $2.8 \%-4 \%$, respectively. The insulin resistance index was calculated from fasting glucose and insulin using the formula of homeostatic model assessment insulin resistance (HOMA-IR). The HOMA index of insulin resistance was calculated as: [insulin (milli international units per liter) x glucose (milli moles per liter)]/22.5.

\section{STATISTICAL ANALYSIS}

The results are expressed as the mean $\pm \mathrm{SD}$. Comparisons of the mean differences in the biochemical parameters between the controls and subjects with diabetes, were performed using student's t-test. The relationships between visfatin with other parameters were explored using the Pearson's correlation technique. Statistical significance is defined as a $p$ value $<0.05$ on two-tailed testing.

\section{RESULTS}

Clinical data of the subjects with diabetes compared to the age-matched controls is shown in Table 1. As expected, subjects with diabetes had higher BMI, higher fasting glucose and increased prevalence of hypertension. Similarly, subjects with diabetes had higher levels of insulin, increased insulin resistance by HOMA-IR and low HDL as shown in Table 2. Subjects with diabetes had significantly lower levels of visfatin compared to the control subjects as shown in Table 3. There was a significant difference in the levels of TNF- $\alpha$ between the two groups as shown in Table 3. Correlation of visfatin to resistin is shown in Figure 1. 


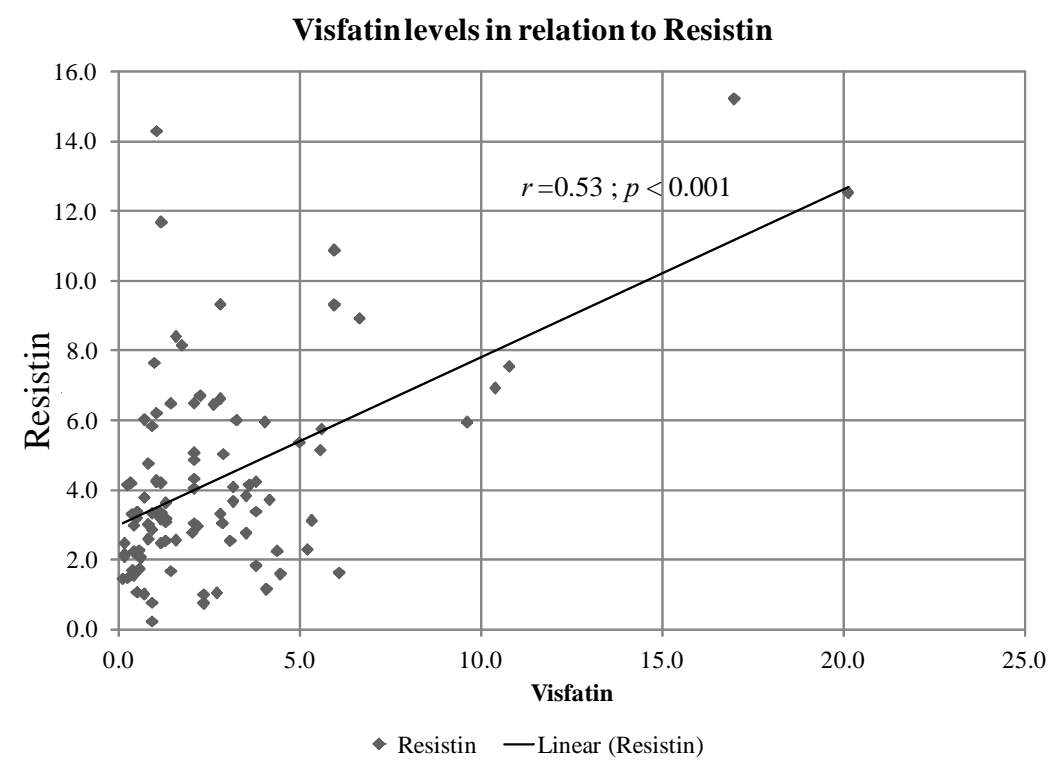

Figure 1. Shows relation of Visfatin to resistin.

Table 3. Adipocyte hormones and inflammatory markers of the subjects with or without diabetes. Data expressed as \pm SD; $p<$ 0.05 is significant; CRP: C-reactive protein; TNF- $\alpha$ : Tumor necrosis factor.

\begin{tabular}{cccc}
\hline & DM & No DM & $\boldsymbol{p}$-value \\
\hline Visfatin & $1.35 \pm 0.2$ & $3.5 \pm 0.47$ & $<0.0001$ \\
Resistin & $3.78 \pm 0.4$ & $4.6 \pm 0.4$ & NS \\
CRP & $4.1 \pm 0.64$ & $2.87 \pm 0.50$ & NS \\
TNF- $\boldsymbol{\alpha}$ & $7.6 \pm 0.78$ & $3.7 \pm 0.35$ & $<0.0001$ \\
\hline
\end{tabular}

\section{DISCUSSION}

There are several significant findings in our study. First, there is a significant difference in the visfatin levels between subjects with and without diabetes. Subjects with diabetes had lower visfatin levels than age-matched controls. This data is similar to the data published by $\mathrm{Li}$ and associates [4]. Esteghamati and associates reported higher levels of visfatin in subjects with diabetes and no association with BMI [5]. Similarly higher levels were reported in other studies $[4,6,7]$. Decreased levels in our subjects might be partly related to pioglitazone use though a short period. In a study by McGee and associates, rosiglitazone decreased visfatin levels significantly along with other cytokines [8]. In contrast, rosiglitazone therapy at $8 \mathrm{mg}$ a day did not affect the visfatin levels in a study of 50 subjects [9].

Resistin and visfatin: In our study, visfatin levels correlate with resistin $(r=0.53 ; p<0.01)$ levels (see Figure 1). Our data is similar to the published literature that visfatin levels correlate with resistin levels [10]. In a review on visfatin and resistin, Stofkova explains how visfatin is proinflammatory and resistin production is increased in inflammatory conditions [11]. The author states that resistin as well as visfatin itself can contribute to the inflammatory processes by triggering cytokine production and NF-kappaB activation [11]. Our results are similar to the results of Samara and associates [12] Plasma levels increase with obesity and correlate positively with visceral adiposity, and visfatin is said to have insulin-mimetic actions, mediated by activation of the insulin receptor in a manner distinct from that of insulin [3].

In a study from the Middle East, Esteghamati and associates [13] reported higher levels of visfatin in subjects with diabetes and positive correlation with insulin and triglycerides, which is exactly opposite of our observations; we noted lower levels of visfatin in subjects with diabetes and negative correlation with insulin and triglyceride levels. Their data being different from ours may be due to difference in the populations. Our data is similar to the published literature in that visfatin levels were lower in subjects with obesity [10].

Combined analysis of all the subjects with and without diabetes, correlation of visfatin levels with CRP was weak $(r=0.12 ; p=0.1)$, but on sub analysis of subjects with diabetes, visfatin correlates with CRP $(r=0.39 ; p<$ 0.01 ). In our study, both in combined analyses as well as sub analyses, the visfatin levels did not correlate with TNF- $\alpha$. In our study, both in combined analyses as well as sub analyses, the visfatin levels negatively correlate with Triglycerides ( $r=0.18 ; p=0.05$ ).

Visfatin (Nampt) has been reported to have an important connection to a wide variety of conditions, including obesity, type 2 diabetes and inflammatory diseases. Reported literature varies regarding the relationship be- 
tween visfatin and diabetes or insulin resistance. There are a number of inconsistencies among the different studies of visfatin, and the role of this adipokine in obesity and insulin resistance is not clear. There is lack of standardization of the assay, which also need to be kept in mind. Studies have clearly demonstrated that Nampt functions as an intra-and extracellular NAD biosynthetic enzyme and that extracellular Nampt/visfatin does not exert insulin-mimetic effects in vitro or in vivo. In addition, visfatin has been shown to exert a number of insulin mimetic and antagonistic effects in cultured adipocytes, hepatocytes and myotubes, and to lower plasma glucose in mice. Plasma visfatin levels were reported to be significantly higher in those with chronic CAD and ACS compared with control patients [14]. Multiple regression analysis demonstrated that plasma visfatin levels correlated with inflammatory factors and were associated with chronic CAD [14]. Zhong and associates also reported that serum visfatin was increased in patients with metabolic syndrome, especially in those with carotid plaques. Visfatin may be an inflammatory marker of metabolic syndrome [15].

In a meta-analysis of the studies of visfatin levels in obesity, type 2 diabetes, insulin resistance, metabolic syndrome and cardiovascular disease, Chang and associates noted inconsistencies in the data sets [16]. They concluded that visfatin may be promising for predicting obesity, diabetes status, insulin resistance, metabolic syndrome and cardiovascular disease with the pooled log odds ratios of visfatin with obesity of 1.164 [95\% confidence interval (CI): 0.348 to $1.981, p=0.005]$, diabetes of 1.981 (95\% CI: 1.377 to $2.584, p<0.001$ ), insulin resistance of 1.094 (95\% CI: 0.678 to $1.511, p<0.001$ ), and cardiovascular disease of 2.902 (95\% CI: 0.924 to 4.879, $p<0.005$ ), respectively [16]. In our study, we noted that the insulin resistance index determined by HOMA-IR did not correlate with visfatin levels.

Several studies now provide supporting evidence that $\mathrm{PBEF} /$ visfatin is primarily a pro-inflammatory cytokine, as its serum/plasma levels have been found to be increased in various inflammatory disorders with association with resistin, TNF- $\alpha$ and CRP $[7,17,18]$. Similar association of visfatin with IL-6 was reported [14]. Similar to our data, the association of visfatin with in- flammatory markers but not insulin resistance was noted by others [19]. All this data indicate that circulating visfatin may reflect inflammation status. The findings in our study are consistent with this hypothesis and confirm the correlation of visfatin with CRP, an inflammatory marker. Studies that look at visfatin levels in relation to atherosclerotic plaques in metabolic syndrome [15] and type 2 diabetes [20] suggested visfatin as a marker of carotid atherosclerosis. In recent review, the authors suggested that research is required to evaluate the real value of visfatin/PBEF/Nampt as a pharmacological target [21].

In conclusion, we noted that circulating visfatin levels is another inflammatory marker and the future awaits visfatin as a pharmacological target.

\section{ACKNOWLEDGEMENTS}

Dr. Yaturu was supported by a VA Merit review grant at the time of the study. Dr. Jain is supported by grants from NIDDK and the Office of Dietary Supplements of the NIH (RO1 DK072433) and the Malcolm Feist Endowed Chair in Diabetes. The authors thank Ms. Barbara Youngberg for excellent editing of this manuscript. None of the authors has any financial interest in publication of this manuscript or has received any money from any other sources than the Veterans Health Administration, NIH or LSUHSC.

\section{REFERENCES}

[1] Samal, B., et al. (1994) Cloning and characterization of the cDNA encoding a novel human pre-B-cell colonyenhancing factor. Molecular and Cellular Biology, 14, 1431-1437.

[2] Luk, T., Malam, Z. and Marshall, J.C. (2008) Pre-B cell colony-enhancing factor (PBEF)/visfatin: A novel mediator of innate immunity. Journal of Leukocyte Biology, 83, 804-816. doi:10.1189/jlb.0807581

[3] Fukuhara, A., et al. (2005) Visfatin: A protein secreted by visceral fat that mimics the effects of insulin. Science, 307, 426-430. doi:10.1126/science. 1097243

[4] Li, L., et al. (2006) Changes and relations of circulating visfatin, apelin, and resistin levels in normal, impaired glucose tolerance, and type 2 diabetic subjects. Experimental and Clinical Endocrinology \& Diabetes, 114, 544548. doi:10.1055/s-2006-948309

[5] Esteghamati, A., et al. (2011) Serum visfatin is associated with type 2 diabetes mellitus independent of insulin resistance and obesity. Diabetes Research and Clinical Practice, 91, 154-158. doi:10.1016/j.diabres.2010.11.003

[6] Filippatos, T.D., et al. (2007) Increased plasma levels of visfatin/pre-B cell colony-enhancing factor in obese and overweight patients with metabolic syndrome. Journal of Endocrinological Investigation, 30, 323-326.

[7] Chen, M.P., et al. (2006) Elevated plasma level of visfatin/pre-B cell colony-enhancing factor in patients with type 2 diabetes mellitus. The Journal of Clinical Endocrinology \& Metabolism, 91, 295-299. doi:10.1210/jc.2005-1475

[8] McGee, K.C., et al. (2011) Visfatin is regulated by rosiglitazone in type 2 diabetes mellitus and influenced by NFkappaB and JNK in human abdominal subcutaneous adipocytes. PLoS One, 6, e20287. doi:10.1371/journal.pone.0020287

[9] Kadoglou, N.P., et al. (2010) Effects of rosiglitazone and metformin treatment on apelin, visfatin, and ghrelin levels in patients with type 2 diabetes mellitus. Metabolism, 59, 373-379. doi:10.1016/j.metabol.2009.08.005 
[10] Straburzynska-Lupa, A., et al. (2010) Visfatin, resistin, hsCRP and insulin resistance in relation to abdominal obesity in women with rheumatoid arthritis. Clinical and Experimental Rheumatology, 28, 19-24.

[11] Stofkova, A. (2010) Resistin and visfatin: Regulators of insulin sensitivity, inflammation and immunity. Endocrine Regulations, 44, 25-36. doi:10.4149/endo $2010 \quad 0125$

[12] Samara, A., et al. (2008) Visfatin, low-grade inflammation and body mass index (BMI). Clinical Endocrinology (Oxford), 69, 568-574. doi:10.1111/j.1365-2265.2008.03205.x

[13] Esteghamati, A., et al. (2012) The value of visfatin in the prediction of metabolic syndrome: A multi-factorial analysis. Journal of Cardiovascular Translational Research, 5, 541-546. doi:10.1007/s12265-012-9373-8

[14] Liu, S.W., et al. (2009) Association of plasma visfatin levels with inflammation, atherosclerosis and acute coronary syndromes (ACS) in humans. Clinical Endocrino$\operatorname{logy}($ Oxford $)$, 71, 202-207. doi:10.1111/j.1365-2265.2008.03453.x

[15] Zhong, M., et al. (2008) Increased serum visfatin in patients with metabolic syndrome and carotid atherosclerosis. Clinical Endocrinology (Oxford), 69, 878-884. doi:10.1111/j.1365-2265.2008.03248.x

[16] Chang, Y.H., et al. (2011) Visfatin in overweight/obesity, type 2 diabetes mellitus, insulin resistance, metabolic syndrome and cardiovascular diseases: A meta-analysis and systemic review. Diabetes Diabetes/Metabolism Research and Reviews, 27, 515-527. doi:10.1002/dmrr.1201

[17] Takebayashi, K., et al. (2007) Association between plasma visfatin and vascular endothelial function in patients with type 2 diabetes mellitus. Metabolism, 56, 451-458. doi:10.1016/j.metabol.2006.12.001

[18] Hammarstedt, A., et al. (2006) Visfatin is an adipokine, but it is not regulated by thiazolidinediones. The Journal of Clinical Endocrinology \& Metabolism, 91, 1181-1184. doi:10.1210/jc.2005-1395

[19] Oki, K., et al. (2007) Circulating visfatin level is correlated with inflammation, but not with insulin resistance. Clinical Endocrinology (Oxford), 67, 796-800. doi:10.1111/j.1365-2265.2007.02966.x

[20] Kadoglou, N.P., et al. (2010) Visfatin (nampt) and ghrelin as novel markers of carotid atherosclerosis in patients with type 2 diabetes. Experimental and Clinical Endocrinology \& Diabetes, 118, 75-80. doi:10.1055/s-0029-1237360

[21] Peiro, C., et al. (2010) Visfatin/PBEF/Nampt: A new cardiovascular target? Frontiers in Cardiovascular and Smooth Muscle Pharmacology, 1, 135. doi:10.3389/fphar.2010.00135 